\title{
A Comparison of Two Input Methods for Keypads on Mobile Devices
}

\author{
Aleks Oniszczak \\ Dept of Computer Science and Engineering \\ York University \\ Toronto, Ontario, Canada M3J 1P3 \\ aleks@cs.yorku.ca
}

\author{
I. Scott MacKenzie \\ Dept of Computer Science and Engineering \\ York University \\ Toronto, Ontario, Canada M3J 1P3 \\ smackenzie@acm.org
}

\begin{abstract}
Two mobile device text entry methods were evaluated. The well-known Multitap method was compared to our RollPad method on a new device utilizing a tactile touchpad in place of a keypad. RollPad was well liked by participants. KSPC (keystrokes per character) was significantly lower: 1.42 compared to 2.13 with Multitap. However, no significant difference was found in error rates or entry speed, with speed measured at about $7.3 \mathrm{wpm}$ for both methods.
\end{abstract}

\section{Keywords}

Tactile feedback, mobile devices, phone keypad, text entry

\section{ACM Classification Keywords}

H.5.2 Information interfaces and presentation (e.g., HCI): User Interfaces---Input devices and strategies, Interaction styles, Auditory (non-speech) feedback, Haptic I/O

\section{INTRODUCTION}

Mobile devices are generally smaller than their non-mobile counterparts and often suffer from compromises due to their size. Most mobile phones use a 12-key keypad, which allows for a small form factor legible labels. Although fine for entering digits, elaborate methods are required to enter alphabetic characters. With the increased demand for mobile text input, better entry methods are desirable.

\section{2-key Text Entry Methods}

There are a few variations on the 12-key keypad, but most are similar to that in Figure 1.

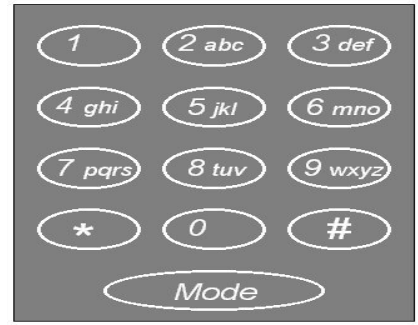

Figure 1. 12-key Keypad Layout (with Mode key)
Early keypads omitted the letters "q" and " $\mathrm{z}$ ", resulting in 3 characters per alpha key. Most current keypads add "q" to the "7" key and " $z$ " to the "9" key (see Figure 1). Occasionally, " $q$ " and " $z$ " are on the " 1 " key. This arrangement is used for the device described herein. With at least 3 characters on each alpha key, a disambiguation method is needed. Some current methods are reviewed.

\section{Multitap}

With Multitap, a desired character is obtained by tapping its key one or more times until it cycles into view. For example, to obtain the character "c", the " 2 " key is pressed quickly 3 times so the device cycles through "a", "b", and then "c". When the desired character is obtained, a time-out or pressing a "time-out key" is required before entering another character on the same key. Problems arise when the user pauses longer than the time-out period while cycling, or does not wait long enough before the next character on the same key. Although widely used, the method remains slow and error-prone [3].

\section{Dictionary Based Disambiguation}

T9 by Tegic Communications (Seattle, WA) uses dictionary-based disambiguation. Similar methods include iTap by Motorola and eZitext by Zi Corp. Users press keys once per character and a dictionary works to match words with key sequences. This works fine when the desired word uniquely appears in the dictionary. Where two words map to the same key sequence (e.g., "cat" and "bat" both map to " 228 "), the device lists the entries ordered by probability. The desired word is selected using a special "next key". If the desired word is not in the dictionary, another input mode is required, such as Multitap.

Two-Key

The Two-key method is not popular, but exemplifies the diverse possibilities of the 12-key keypad. It involves first pressing a key to choose a character group, then pressing 1, 2,3 , or 4 to select a character within the group.

Less-Tap [3]

Less-Tap is a variation on Multitap wherein the letters on each key are ordered according to their frequency in the language. For English, the " 2 " key cycles the characters in order "acb" rather than "abc", because "c" appears more often than "b" [3]. Although fewer keystrokes are required, attention demands are higher since the user must attend to the display to observe the effect of each key press. 


\section{TiltText [5]}

In TiltText, tilting the phone in one of four directions results in the selection of a character associated with the direction and key pressed. For example, to obtain an "a", the phone is tilted to the left and " 2 " is pressed. A similar procedure while tilting right produces "c", etc. TiltText results in faster input than the MultiTap method [5]

\section{LetterWise}

LetterWise (Eatoni Ergonomics, New York) uses prefixbased disambiguation utilizing a database of letter sequences and their probabilities of appearing in the language. The most-probable letter is presented based on previously entered characters. If the presented letter is not correct, the user presses the "next key" until the desired letter appears. An advantage over T9 is that both dictionary and non-dictionary words are entered with similar success. However, attention demands are high, since the result of each key press must be monitored.

\section{Keypad Types}

There are two main types of 12-key keypads in use. The traditional keypad employs physical buttons with tactile and aural feedback. A newer method is virtual keypads, which display simulated buttons on a small screen. Virtual keypads are found where size constraints preclude physical keypads. The methods above can be implemented on either type of keypad. However, touch-sensitive displays lack innate tactile and aural feedback which adversely affects speed and throughput [1]. To address this, adding hardware such as a piezzo device under a touch screen have been successfully demonstrated [4].

\section{RollPad}

RollPad is a new method of inputting characters onto a 12key soft keypad. While soft keypads are generally implemented as a compromise, RollPad leverages a property of finger-activated touchscreens and touchpads that physical buttons lack - the ability to be "rolled". For example, to input the letter "f", a press of the " 3 " key with a slight rolling motion of the finger to the right produces the desired character. The direction of the roll corresponds to the position of the letter in the triad. So, pressing " 3 " with a left roll produces "d", pressing " 3 " with no roll produces the middle "e", and pressing " 3 " with a right roll produces the rightmost character, "f". Keys 2-9 operate in this way while key " 1 ", produces "q" with a leftward roll and a "z" with a rightward roll. See Figure 2.

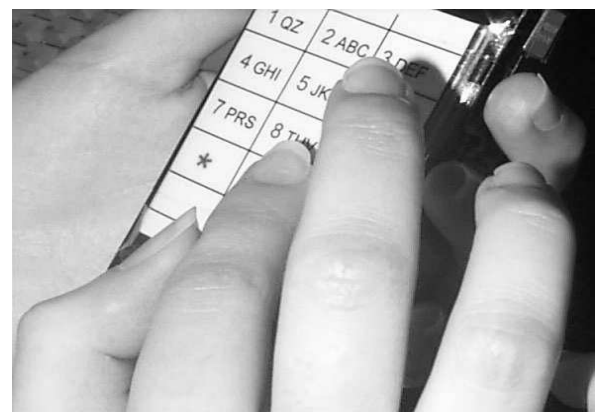

Figure 2. The RollPad in Use
Some implementations of soft keyboards involve a steep learning curve [2]. To further improve speed and accuracy of the device and minimize additional learning, aural and tactile feedback was added as was done with our earlier Tactile Touchpad [1]. In the following section we present an evaluation of RollPad. A touch-based implementation of Multitap was included as a point of comparison.

\section{METHODOLOGY}

\section{Participants}

Fourteen participants ( 8 male, 6 female) from the local student community were recruited for the study. Participants ranged in age from 18 to 35 (mean $=25.7, s d=$ 5.8). Eight described themselves as experienced with Multitap while the remaining six reported no prior experience with any mobile device text entry technique.

\section{Apparatus}

A $1 \mathrm{GHz}$ Celeron computer with 17" monitor running Windows Millennium Edition was used with experiment software written in Java 2 (V1.4). The prototype used a Versapad 80-10032B serial touchpad (Interlink Electronics, Camarillo, CA), which was overlaid on the casing of a small Panasonic KX-T4001BH cordless handset (Figure 3). Inside the device were installed two OEG OUA-SS-105D $5 \mathrm{~V}$ relays to produce localized tactile and aural feedback [1] (Figure 3). A 16x2 line Crystal Control 632 USB LCD panel was attached to the unit to provide visual feedback of the entered text. Power was supplied by the keyboard port on the PC. The serial touchpad and USB display derived their power through their respective ports. Communication with the relays utilized the PC's parallel port.

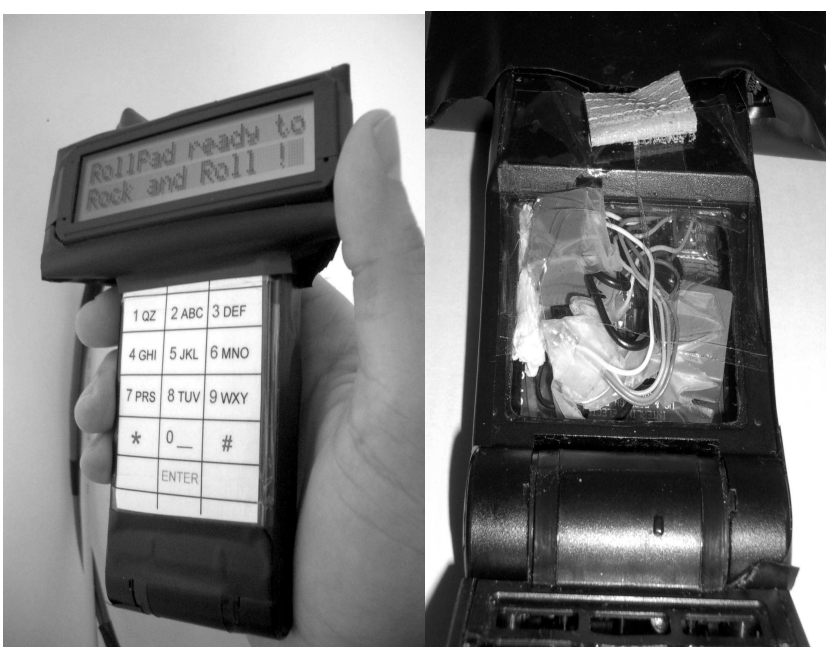

Figure 3. Front and Internal Views of Device

For each trial, a short phrase was displayed on the PC monitor. Phrases were selected at random from a set of 500 phrases, ranging in length from 16 to 43 characters.

\section{Procedure}

The experiment was performed in a quiet campus lab. Participants sat at a desk with the apparatus in front of them (Figure 4). Both methods utilized the same input device. The experimenter explained the tasks and demonstrated the 
procedure prior to data collection. Participants were instructed to enter the presented phrases onto the device until a tone signaled them to stop. Two 12-minute blocks of trials were given for each method. Participants practiced entering two sentences prior to starting each method. The forefinger of the dominant hand was used for input, with the device held in the non-dominant hand. Measurements such as entry speed and number of errors were recorded with the first keystroke of each phrase and ended when the ENTER key was pressed.

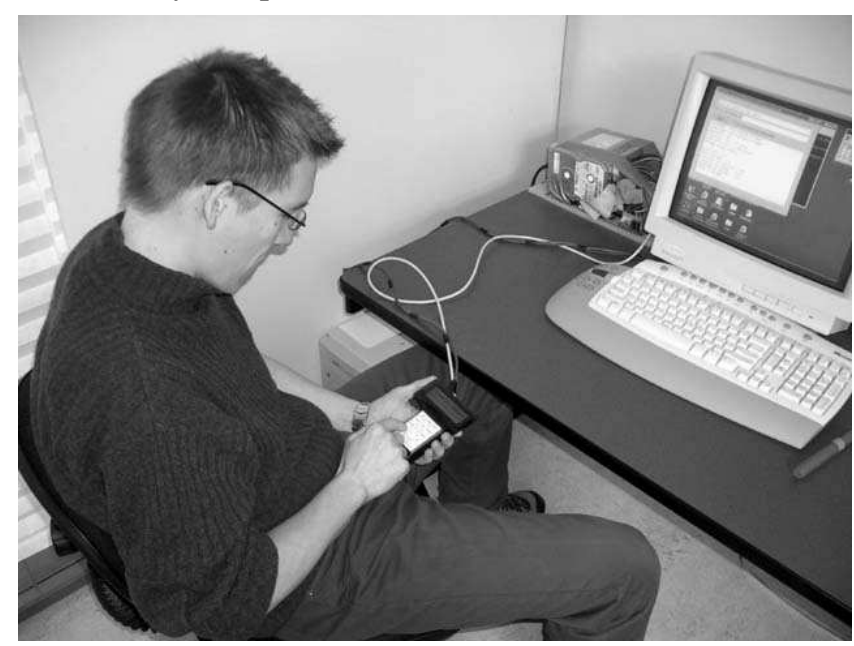

Figure 4. Experimental Environment

\section{Design}

The experiment was a 2 × 2 within subjects design. Each of participant performed two blocks for each of two methods. Half the participants started with the RollPad method; half with Multitap.

\section{RESULTS AND DISCUSSION}

\section{Speed and Accuracy}

There was no significant difference in entry speed, measured in words per minute, between the Rollpad and Multitap methods $\left(F_{1,13}=0.04, n s\right)$. Figure 5 shows that the rate for Rollpad averaged $7.32 \mathrm{wpm}$ while Multitap averaged $7.33 \mathrm{wpm}$.

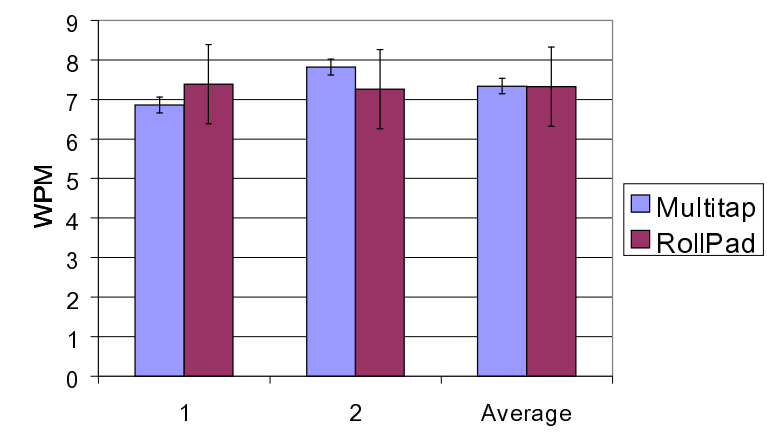

Figure 5. Words Per Minute

There was also no significant difference in entry speed by block. The range was from 6.85 to $7.82 \mathrm{wpm}$, which is typical for novice users of mobile phones with standard keypads [3]. Interestingly, when only the eight expert participants' data were analyzed, there was still no significant difference between methods $\left(F_{1,7}=0.64, \mathrm{~ns}\right)$. This possibly suggests that skills are somewhat transferable from MultiTap to RollPad. Informal observation revealed that familiarity with the keypad layout was noticeably different between expert and novice users: even though the characters were laid out alphabetically (with the exception of the infrequently used "q" and " $z$ " keys), expert users were quicker to find the desired keys.

There was also no significant difference in error rates between the two methods $\left(F_{1,13}=0.75, \mathrm{~ns}\right)$, as illustrated in Figure 6. Again, there was no significant difference by block either. The MultiTap condition had $7.63 \%$ errors and the RollPad condition had $6.97 \%$ errors.

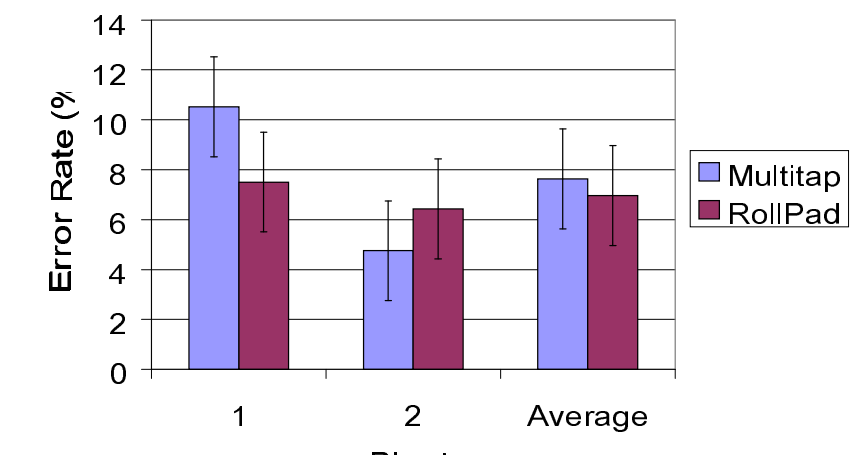

Figure 6. Error Rate

\section{Keystrokes Per Character (KSPC)}

Unsurprisingly, there was a highly significant difference found between methods in KSPC (keystrokes per character $\left(F_{1,13}=245.18, p<.0001\right)$, as seen in Figure 7 .

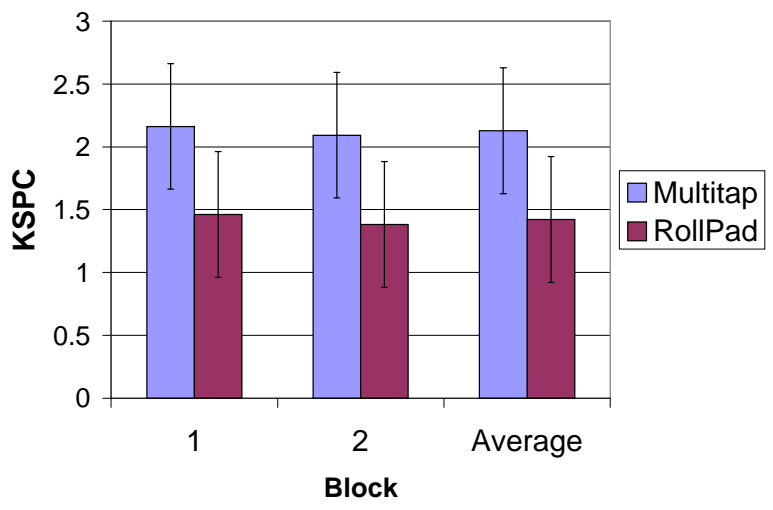

Figure 7. Keystrokes Per Character

Since theoretically the RollPad technique requires only one keystroke per character, and Multitap requires 2.0342 [6], this is expected. In the experiment, RollPad scored 1.42 compared to 2.13 with MultiTap. However, given this innate advantage, the preceding findings of no significant difference in speed or accuracy between the techniques suggest there is at least one factor hindering the RollPad 
method. There may be a greater cognitive load required in rolling one's fingers in the proper direction or perhaps rolling simply takes longer than the equivalent number of taps in the Multitap technique. Further study is warranted. Counterbalancing the order of testing layouts achieved the desired result as the main effect and interactions for Group were not statistically significant.

There was a significant gender difference in entry speed $\left(F_{1,13}=12.12, p<.005\right)$, accuracy $\left(F_{1,13}=17.33, p<.005\right)$, and $\operatorname{KSPC}\left(F_{1,13}=162.5, p<.005\right)$, with males entering text faster, more accurately, and with less keystrokes than females. Differences between the methods within each gender were investigated. No significant differences were found in entry speed $\left(F_{1,7}=0.61, \mathrm{~ns}\right)$ for males and $\left(F_{1,5}=\right.$ $0.96, \mathrm{~ns})$ for females or accuracy $\left(F_{1,7}=3.08, p>.05\right)$ for males and $\left(F_{1,5}=0.04, \mathrm{~ns}\right)$ for females.

Next, a difference between methods among the expert users was tested; however, none was found $\left(F_{1,7}=0.30, \mathrm{~ns}\right)$ for entry speed or error rate $\left(F_{1,7}=3.32, p>.05\right)$. Also, none was found among inexperienced users in entry speed $\left(\mathrm{F}_{1,5}=\right.$ $0.64, \mathrm{~ns})$ or error rate $\left(F_{1,5}=0.12, \mathrm{~ns}\right)$. It appears that the methods are neither better nor worse than each other.

\section{Tactile Feedback}

One interesting observation was that most participants accepted the tactile and aural "clicking" of the device as natural. It was only when the effect was turned off and they could no longer "feel" the buttons did they express surprise. Also surprising was that no participant suggested they would have preferred a keypad with physical buttons, not even in the MultiTap method. As the speed and accuracy were comparable to that of what users attain with traditional mobile phones [3], this suggests that virtual keypads can be functionally equivalent to physical keypads.

\section{Critiquing the Method}

The present evaluation assessed only initial user performance, and did not address prolonged use. One of the authors who practiced extensively scored almost $16 \mathrm{wpm}$ with RollPad while attaining only 9 wpm using Multitap. The peak was over $21 \mathrm{wpm}$ with no errors. A long-term study with more participants would prove useful to investigate the possibility that RollPad becomes faster than Multitap with extended practice.

Phrases used in this study are typical of language used in email correspondence and have a high correlation with existing language corpora. However, they are not representative of typical phraseology in mobile phone text messages. Users tend to shorten their words and phrases to minimize key presses. This behavior has resulted in a slang unique to text messaging (e.g., "are you there" becomes " $r$ u thr"). However, since the same phrases were used in each method, the advantages and limitations of entering standard English affected both methods. Whether it affected one method more than the other is a topic for further investigation.
Further study could also investigate whether a variation on RollPad could work with a more standard keypad layout, for example, rolling downward for the fourth letter on a key. A similar technique has been previously tested [5].

\section{Participant Questionnaire}

Participants completed a post-test questionnaire. In it, Multitap was rated quicker (9:5), less error prone (10:4) and easier to learn (9:5). Participants were split as to which felt more fatiguing and, interestingly, RollPad was slightly preferred overall (8:6). Given the perception of the RollPad being slower and more error prone, it was surprising that it was better liked. This may be due to participants wanting to please the experimenter. However, comments reveal a "coolness" factor to RollPad not ascribed to Multitap. Comments such as "I like the flow in roll top" [sic] and "Really wicked" attest to this.

\section{CONCLUSION}

Two techniques for text entry on mobile devices were evaluated. MultiTap technique utilized the standard method of input used on mobile phones today. RollPad uses one touch per key, combined with a rolling action to select the desired letter. KSPC (keystrokes per character) differed significantly different between RollPad (1.42) and Multitap (2.13). However, no significant differences were found in text entry speed or accuracy. RollPad averaged $7.32 \mathrm{wpm}$ while Multitap averaged $7.33 \mathrm{wpm}$.

Participants generally liked the novel RollPad technique and accepted the unusual addition of tactile feedback to the device. Further research is warranted in evaluating the two methods in a longitudinal study.

\section{ACKNOWLEDGMENTS}

Thanks go to Interlink Electronics for providing the touchpad and technical documentation for this experiment.

\section{REFERENCES}

1. MacKenzie, I. S., \& Oniszczak, A. A comparison of three selection techniques for Touchpads. Proc CHI'98, 336-343.

2. MacKenzie, I. S., \& Zhang, S. X. The design and evaluation of a high-performance soft keyboard. Proc CHI '99, 25-31.

3. Pavlovych, A., \& Stuerzlinger, W. Model for nonexpert text entry speed on 12-button phone keypads. Proc CHI '04, 351-358

4. Poupyrev, I., Maruyama, S., \& Rekimoto, J. TouchEngine: A tactile display for handheld devices. Proc CHI '02, 644-645.

5. Wigdor, D., \& Balakrishnan, R. TiltText: Using tilt for text input to mobile phones, Proc UIST '03, 81-90.

6. MacKenzie, I. S. KSPC (keystrokes per character) as a characteristic of text entry techniques. Proc 4th Int Symp HCI with Mobile Devices. 195-210. 